\title{
Homoclinicity in the dynamics of forced fluidized bed catalytic reactors
}

\begin{abstract}
The dynamic behaviour of the non-isothermal oscillating fluidized bed catalytic reactor subjected to high and low frequency forcing is investigated. Resonance horns and period doubling loci inside the horns are constructed, using efficient algorithms, for different positions of the centre of forcing relative to the homoclinical orbit (infinite period orbit) of the autonomous (unforced) system. The analysis shows that the reactor behaviour is very sensitive to the position of the centre of forcing relative to the homoclinical orbit. For high frequency forcing $(\gamma / \gamma 0=5 / 1)$ with centre of forcing close to the homoclinical orbit, the system shows period doubling to chaos at very small amplitudes. For low frequency forcing $(\gamma / \gamma 0=2 / 1)$, incomplete period doubling not leading to chaos is observed in some cases and the new period adding bifurcation is also uncovered for other cases. The interactions between different resonance horns are investigated and the resulting bistabilities are elucidated. Quantitative methods proved that the strange attractors encountered are chaotic with one positive Lyapunov exponent and a Lyapunov fractal dimension between 1 and 2.
\end{abstract}

Keyword: Fluidized bed catalytic reactor; Homoclinicity; Resonance horns; High and low frequency forcing 